\title{
A Ferret Model of Encephalopathy of Prematurity
}

\author{
Thomas Wood $^{\text {a }}$ Daniel Moralejo ${ }^{a}$ Kylie Corry $^{\text {a }}$ Jessica M. Snyder ${ }^{b}$ \\ Christopher Traudt ${ }^{a}$ Chad Curtis $^{c}$ Elizabeth Nance ${ }^{c}$ Pratik Parikh ${ }^{a}$ \\ Sandra E. Juul ${ }^{a}$ \\ ${ }^{a}$ Division of Neonatology, Department of Pediatrics, University of Washington, Seattle, WA, USA; ${ }^{b}$ Department \\ of Comparative Medicine, University of Washington, Seattle, WA, USA; 'Department of Chemical Engineering, \\ University of Washington, Seattle, WA, USA
}

\section{Keywords}

Prematurity · White-matter injury · Development · Brain .

Neonatal brain injury

\begin{abstract}
There is an ongoing need for relevant animal models in which to test therapeutic interventions for infants with neurological sequelae of prematurity. The ferret is an attractive model species as it has a gyrified brain with a white-to-gray matter ratio similar to that in the human brain. A model of encephalopathy of prematurity was developed in postnatal day 10 (P10) ferret kits, considered to be developmentally equivalent to infants of 24-26 weeks' gestation. Cross-fostered P10 ferret kits received $5 \mathrm{mg} / \mathrm{kg}$ of lipopolysaccharide (LPS) before undergoing consecutive hypoxia-hyperoxiahypoxia (60 $\mathrm{min}$ at $9 \%, 120 \mathrm{~min}$ at $60 \%$, and $30 \mathrm{~min}$ at $9 \%$ ). Control animals received saline vehicle followed by normoxia. The development of basic reflexes (negative geotaxis, cliff aversion, and righting) as well as gait coordination on an automated catwalk were assessed between P28 and P70, followed by ex vivo magnetic resonance imaging (MRI) and immunohistochemical analysis. Compared to controls, injured animals had slower overall reflex development between P28
\end{abstract}

and P40, as well as smaller hind-paw areas consistent with "toe walking" at P42. Injured animals also displayed significantly greater lateral movement during CatWalk assessment as a result of reduced gait coordination. Ex vivo MRI showed widespread white-matter hyperintensity on T2-weighted imaging as well as altered connectivity patterns. This coincided with white-matter dysmaturation characterized by increased intensity of myelin basic protein staining, whitematter thinning, and loss of oligodendrocyte transcription factor 2 (OLIG2)-positive cells. These results suggest both pathological and motor deficits consistent with premature white-matter injury. This newborn ferret model can therefore provide an additional platform to assess potential therapies before translation to human clinical trials.

๑) 2019 S. Karger AG, Basel

\section{Introduction}

There is an ongoing need for large-animal models that reflect the pathophysiology of preterm brain injury in which therapeutic interventions for infants with neurological sequelae of prematurity can be tested. In 2016, $9.85 \%$ of infants born in the USA were premature, and,

\section{KARGER}

(c) 2019 S. Karger AG, Basel

E-Mail karger@karger.com

www.karger.com/dne
Dr. Thomas Wood, BM, BCh, PhD

Division of Neonatology, Department of Pediatrics

University of Washington Medical Center, Box 356320, HSB RR-535

Seattle, WA 98195 (USA)

E-Mail tommyrw@uw.edu 
for those born extremely preterm ( $<28$ weeks' gestation), around $50 \%$ will have a poor outcome [1-3]. Preterm birth is commonly initiated by maternal infection (chorioamnionitis) and is also often associated with perinatal insults such as hypoxia and ischemia [4]. Subsequent intermittent iatrogenic hyperoxia is commonplace in the neonatal intensive care unit (NICU) [5]. These combined factors are thought to contribute to developmental and physiologic vulnerability of the brain, and to result in or exacerbate the encephalopathy associated with poor developmental outcomes in preterm infants [5-7].

Recently, because of the physical and developmental similarities in the ferret and human brain, the ferret has emerged as an attractive species in which to model brain injury [8-11]. Unlike rodents and rabbits, the ferret has a gyrencephalic cerebral cortex and a ratio of white-to-gray matter that is similar to humans. Ferrets are ideal candidates to model preterm brain injury as they are born lissencephalic, developing gyrencephalic brains postnatally. Postnatal white-matter maturation and complex cortical folding in ferret kits occur in a similar pattern to that observed in the human brain during the third trimester [12]. This includes development of the cortical subplate (a transient scaffolding for the evolving cortex), prominent in human brain development but minimal in rodents [13]. At birth, ferret brain development is similar to that of a 13-week-old human fetus, with postnatal day 10 (P10) kits considered to be equivalent to an infant of 2426 weeks' gestation [12].

We have previously examined the white matter and motor development in the newborn ferret, with preliminary data suggesting that a lipopolysaccharide (LPS)-sensitized hypoxic and hyperoxic insult could result in shortterm inflammation in the P10 ferret brain, including the activation of microglia and possible astrogliosis [11]. Here, we describe the long-term behavioral and pathological outcomes of inflammation-sensitized hypoxic/hyperoxic brain injury in the P10 ferret as a model of encephalopathy of prematurity.

\section{Methods}

\section{Animals}

Eight time-mated pregnant jills were acquired from Marshall BioResources (North Rose, NY, USA) at $\leq 28$ days of gestation, and allowed to kindle naturally (typical gestation 41-42 days). Pregnant jills were acquired in pairs, and kits were cross-fostered at P8/ P9 in order to balance litter sizes and sex distribution. Animals were maintained in a centralized vivarium with ad libitum access to food and water before and during experimental procedures. Standard housing ferret conditions included a 16-h light/8-h dark cycle with a room temperature (RT) range of $61-72^{\circ} \mathrm{F}\left(16-22^{\circ} \mathrm{C}\right)$, humidity of $30-70 \%$, and $10-15$ fresh air changes per hour.

\section{Experimental Procedure}

On P10, cross-fostered ferret kits were randomized to receive an intraperitoneal $5 \mathrm{mg} / \mathrm{kg}$ dose of LPS (from Escherichia coli O111:B4, List Biological, CA, USA) or saline vehicle, before being returned to their jills for $4 \mathrm{~h}$. LPS-injected animals were subsequently placed in a humidified chamber within a water bath, and underwent consecutive hypoxia, hyperoxia, and hypoxia (60 min at $9 \% \mathrm{O}_{2}, 120 \mathrm{~min}$ at $60 \% \mathrm{O}_{2}$, and $30 \mathrm{~min}$ at $9 \% \mathrm{O}_{2}$ ). Rectal temperature in a sentinel animal was monitored continuously throughout the insult (Precision 4000A thermometer, YSI, Yellow Springs, OH, USA), with a target intrahypoxic rectal temperature of $37^{\circ} \mathrm{C}$. Saline controls received an identical period of normoxia, after which all animals were returned to their jills. A number of experimental protocols that did not produce any evidence of short-term injury were attempted in the development of the current approach. A summary of these is listed in online supplemental Table 1 (for all online suppl. material, see www.karger.com/ doi/10.1159/000498968). The final protocol was developed based on this preliminary data as well as on evidence from the preclinical literature. An LPS dose of $5 \mathrm{mg} / \mathrm{kg}$ was chosen because preliminary experiments (online suppl. Table 1 ) showed greater histological injury in kits exposed to 3 doses of $5 \mathrm{mg} / \mathrm{kg}$ LPS before hypoxia/hyperoxia compared to doses of $2 \mathrm{mg} / \mathrm{kg}$ LPS, but the 5 $\mathrm{mg} / \mathrm{kg}$ dose was similar to $10 \mathrm{mg} / \mathrm{kg}$ (online suppl. Fig. 1). A single LPS dose was then chosen due to concerns about potential preconditioning caused by multiple doses [11]. A 4-h delay between LPS exposure and hypoxia was used based on rodent literature, as this is the time frame associated with the greatest systemic inflammatory response and sensitization of the brain to hypoxia-ischemia [14-16]. A similar time course of inflammatory activation after LPS exposure has been shown in isolated ferret peripheral blood mononuclear cells [17]. The resulting final protocol published here was the first to show sustained pathological and neurobehavioral deficits in exposed P10 ferret kits. One iteration of the protocol ( 3 doses of LPS every $12 \mathrm{~h}$ at P9/P10 before $45 \mathrm{~min}$ at $6 \%$ oxygen and $6 \mathrm{~h}$ at $100 \%$ oxygen) was included in a previous publication [11].

\section{Early Behavioral Testing}

Beginning on P28, kits underwent reflex testing 3 times/week until P40. This included negative geotaxis (NG), cliff aversion (CA), and righting reflex (RR), as previously described [11]. Briefly, NG was performed on inclined planes at $45^{\circ}$. Kits were placed head-down in the middle of the plane, and time taken to rotate $90^{\circ}$ and $180^{\circ}$ was recorded. For CA, kits were placed with both front paws on the edge of a shelf. Time to spontaneously retreat away from the edge and rotate away was recorded. For RR, animals were placed on their backs and time to righting was recorded; they were required to show full-paw placement and begin coordinated locomotion to complete the righting task. A total of 3 runs of the NG and CA tests and 5 runs of the RR test were performed on every testing day. For all tests, failure was considered to be an inability to complete the task within $60 \mathrm{~s}$. Total time (TT) score, i.e., the sum of the mean time for each of the 3 tasks on each day and area under the curve (AUC) across the whole testing period (as a measure of skill development over time) for each test were also calculated. 
CatWalk Analysis

From P42 to P70, kits underwent weekly catwalk gait analysis (CatWalk XT, Noldus, Leesburg, VA, USA). At each time point, 3 compliant runs (lasting $<10 \mathrm{~s}$ with a maximum speed variation of $60 \%$ ) were collected from each animal. Paw placement pressure, paw area, stride length, swing speed, and base of support (BOS) were analyzed over time as measures of gait development. Due to variability in size between both litters and sexes, all CatWalk measures were either adjusted by weight, or used to generate ratios (i.e., between the forepaws and hind paws) within a single animal. Measures of paw pressure intensity were derived from CatWalk XT and are expressed in arbitrary units (au). A Python package was developed to analyze the lateral movement component of ferrets during the catwalk task. Raw images were extracted from the CatWalk dataset for postanalysis with a self-developed software package and an ImageJ macro (https://github.com/ccurtis7/ferretfit). Paw print coordinates were extracted from raw output images. Trajectory features were extracted from each run that gave measures of the lateral spread of the paw prints, including range and standard deviation in the lateral direction as well as the amplitude and period of a sinusoidal curve fitted to the trajectory of the animal's path (both measured in pixels).

\section{Ex vivo Magnetic Resonance Imaging and Network \\ Connectivity}

At P70, kits underwent euthanasia and perfusion fixation with PBS followed by $10 \%$ neutral buffered formalin (NBF). Subsequently, the brains were removed and immersion-fixed in NBF for at least a further $72 \mathrm{~h}$, before being rinsed and submerged in PBS at $4^{\circ} \mathrm{C}$ to rehydrate for $72 \mathrm{~h}$. Brains were then mounted on agarose gel sleds inside $50-\mathrm{mL}$ Falcon tubes and immersed in Fomblin (perfluoropolyether [PFPE]; Solvay Specialty Polymers, GA, USA). Diffusion-weighted magnetic resonance imaging (MRI) data were collected on the Bruker Avance III, 4.7-tesla $\left(200 \mathrm{MHz},{ }^{1} \mathrm{H}\right), 20-\mathrm{cm}$ horizontal-bore magnet with ParaVision v6.0.1. The magnet is fitted with custom (Resonance Research, Billerica, MA, USA), 9-cm i.d., high-performance gradients achieving an average of 750 $\mathrm{mT} / \mathrm{m}$ gradient with a $100-\mu$ s slew rate. A TurboRARE 3 -dimensional (3D) T2 sequence provided anatomical images using the following settings: field-of-view (FOV) $30 \mathrm{~mm}$, slice thickness 0.23 $\mathrm{mm}$, sagittal-slice orientation matrix with a 1.6-s recycle time and 39-ms echo time accomplished via a rare factor of 16 and acquired into a 128 points per axis cubic volume. Diffusion tensor images (DTIs) were obtained using a $2 \mathrm{D}$ collection with the same FOV as the T2. Slice thickness was $0.6 \mathrm{~mm}$ for 30 diffusion directions (plus 5 A0 images), and 8 averages for 64 slices $(128 \times 128)$ each with a thickness of $0.6 \mathrm{~mm}$. The recycle time was set to $5.6 \mathrm{~s}$ with an echo time of $48 \mathrm{~ms}$. Diffusion weighting was set to a 4 -ms gradient duration $(\delta)$ and 10 -ms gradient separation $(\Delta)$ with a maximum $B$ value of 4,320 to result in 30 diffusion directions. Diffusionweighted images were motion- and distortion-corrected using the latest version of FMRIB Software Library (FSL v5.0, Oxford, UK) eddy software (https://fsl.fmrib.ox.ac.uk/fsl/fslwiki/eddy). FSL's dtifit was then applied, and the resulting DTI median was filtered using the fslmaths-fmedian option. The resulting output files were fractional anisotropy (FA), L1, L2, L3 (the 3 eigenvalue maps), MD (mean diffusivity), and MO (dti mode) maps. Radial diffusivity was calculated by combining the L2 and L3 maps. Coregistration of the FA maps from all subjects was performed using Tract-Based Spatial Statistics (TBSS). This procedure builds a template from all subjects and coregisters individual FA maps to the same template. Comparisons of TBSS between groups were threshold-free cluster enhancement (TFCE) adjusted for multiple comparisons. DTI connectivity values were measured using FSL's probtrackx2 software with the network option enabled and using 71 different anatomical seed points and associated regions of interest (ROIs) spread throughout the brain. Matlab "Brain Connectivity Toolbox" (https:// sites.google.com/site/bctnet/construction) was used to perform the complex network analysis/graph theory analysis, as described by Rubinov and Sporns [18]. The clustering coefficients of the regions were based on the full-brain connectivity network regions thresholded at $10 \%$ sparsity. Graph-based network analysis allows for the visualization of connectivity patterns among all the elements of the brain (e.g., brain regions) as well as the quantitative characterization of global organization. The utility of graph-based techniques has been proven by an increasing number of studies probing potential mechanisms involved in normal development [19-21], aging [22, 23], and various brain disorders [24-26].

\section{Immunohistochemistry}

After performing MRI, coronal slices at the level of the caudate nucleus were taken from each brain, embedded in paraffin, and $4-\mu \mathrm{m}$ sections were prepared for hematoxylin and eosin (H\&E) staining and immunohistochemistry (IHC). Myelin basic protein (MBP) and oligodendrocyte transcription factor 2 (OLIG2) IHC was performed at the University of Washington Histology and Imaging Core. For MBP, slides were baked for 30 min at $60^{\circ} \mathrm{C}$ and deparaffinized on the Leica Bond automated immunostainer (Leica Microsystems, Buffalo Grove, IL, USA). Antigen retrieval was performed by placing slides in EDTA for $20 \mathrm{~min}$ at $100^{\circ} \mathrm{C}$. The primary antibody (rat anti-ferret MBP, 1:500 dilution, Abcam, AB7349) in Leica primary antibody diluent was applied for $30 \mathrm{~min}$. A secondary antibody, unconjugated rabbit anti-rat IgG $(1: 300+5 \%$ NGS in TBS, Vector, AI-4001) was then applied for $8 \mathrm{~min}$. Goat anti-rabbit horseradish peroxidase Leica Bond polymer was added for 8 min. Antibody complexes were visualized using Leica Bond mixed refine (DAB, $3,3^{\prime}$-diaminobenzidine) detection $2 \times$ for $10 \mathrm{~min}$ at RT. For OLIG2, staining was performed using rabbit polyclonal antiOLIG2 (Millipore, cat. No. AB9610.) on formalin-fixed paraffinembedded sections. Antigen retrieval was performed by placing slides in citrate for $20 \mathrm{~min}$ at $100^{\circ} \mathrm{C}$. The primary antibody, OLIG2 $(1: 500)$ or rabbit IgG $(1: 1,000)$ in Leica primary antibody diluent, was applied for $30 \mathrm{~min}$ at RT. In both the MBP and OLIG2 protocols, tissues were counterstained with hematoxylin for $4 \mathrm{~min}$. GFAP IHC was performed at the University of Washington Harborview Medical Center histology IHC lab. For GFAP, staining was performed using rabbit polyclonal antiglial fibrillary acidic protein (GFAP; Agilent, Dako) at a dilution of 1:300, using the Leica Bond III IHC stainer, the polymer refine detection kit, and the Bond epitope retrieval 1 solution for $20 \mathrm{~min}$. These antibodies had not previously been optimized in the ferret. Images of representative brain regions were acquired from digitally scanned images or glass slides using NIS-Elements BR 3.2 64-bit and plated in Adobe Photoshop Elements. Image brightness and contrast were adjusted using auto smart fix and/or auto white balance, with manipulations applied to the entire image. The original magnification and/or scale bar is stated. 
Fig. 1. Weight gain. On the day of the insult (P10), mean (SD) weight of the kits was 41.4 (8.8) $\mathrm{g}$ for males and 38.8 (7.2) $\mathrm{g}$ for females. Injured animals lost, on average, $8.5 \%$ (10.4\%) body weight between P10 and $\mathrm{P} 11$ as a result of the insult. By P12, injured animals had gained $9.2 \%(13.7 \%)$ of their P10 body weight, compared to a $24.4 \%$ (5.2\%) weight gain in controls at P12. By the 6th week of age (P35-P42), injured animals had caught up in terms of weight to control animals, and males began to become heavier than females. No difference in weight between injured and control animals was seen at P70.

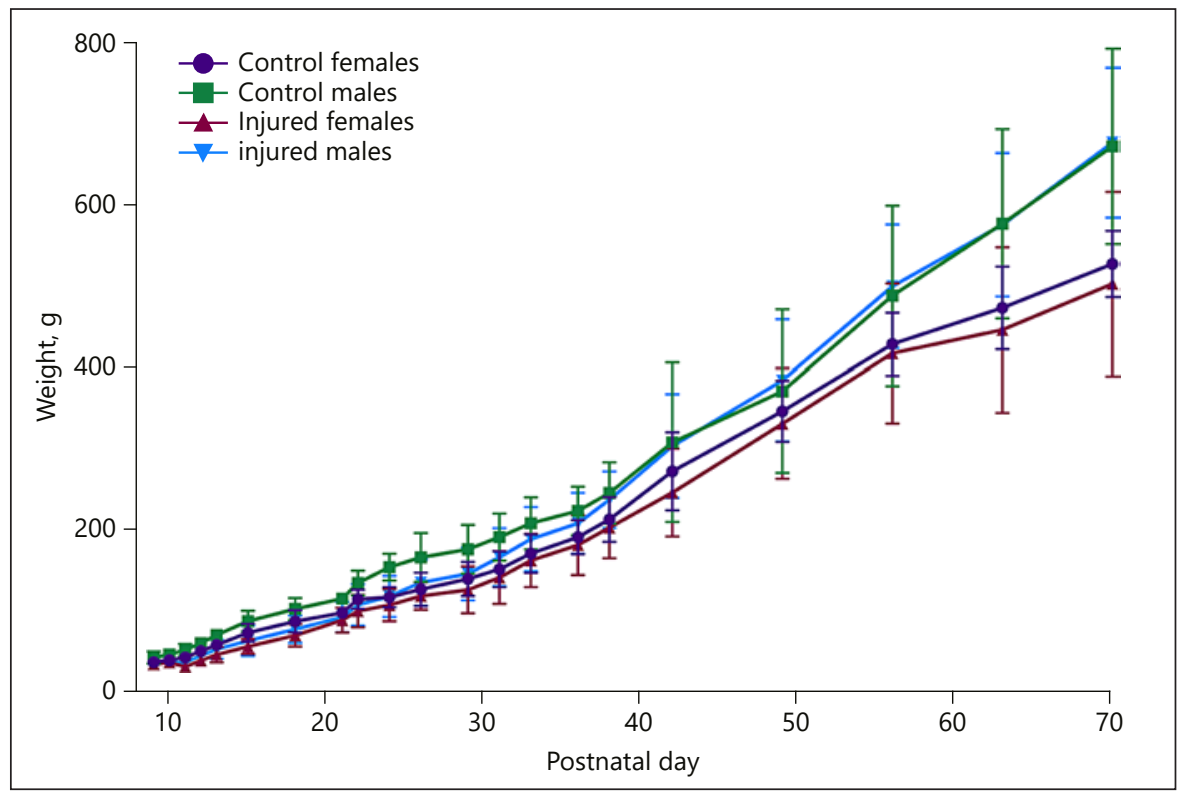

\section{Quantitative IHC}

Image analysis was performed using whole-slide digital images and automated image analysis. All slides were scanned in bright field with a $\times 20$ objective using a Nanozoomer Digital Pathology slide scanner (Hamamatsu; Bridgewater, NJ, USA). Whole-slide digital images were imported into Visiopharm software (Hoersholm, Denmark) for analysis. The software converted the initial digital imaging into gray-scale values using 2 features, RGB-R with a mean filter of $5 \times 5$ pixels and an RGB-B feature. Visiopharm was then trained to label positive staining and the background tissue counterstain using a project-specific configuration based on threshold pixel values. Images were processed in batch mode using this configuration to generate the desired outputs (Ex. area of MBP and ratio of MBP to total tissue area). For quantitative measurements of MBP, OLIG2, and GFAP, the Visiopharm image analysis module was used to define ROIs. ROIs were selected by manually drawing around or placing a circle of equal size within the anatomic structure of interest. ROIs were selected, in part, based on areas noted to be potentially different between treated and control animals on MRI; they included half of the brain, a circular region of the corpus callosum (CC), a circular region of the dorsal internal capsule (IC), a circular level of the ventral IC, and the dorsal cerebrum (online suppl. Fig. 2). As described above, positively stained versus unstained tissue was segmented using a project-specific configuration to generate the desired outputs. After quantitative staining analysis, MBP images were imported into NDP. view2 (Hamamatsu Photonics, Bridgewater, NJ, USA), and the thickness of the CC and 3 levels of the IC (online suppl. Fig. 2) were measured by an operator blinded to the treatment groups.

\section{Statistical Analysis}

Statistics and images were generated in GraphPad Prism v7 (San Diego, CA, USA). Early reflex testing was analyzed by comparing the injured and control groups at each time point using a
Wilcoxon-Mann-Whitney U-test. CatWalk data were primarily compared between groups at $\mathrm{P} 42$, as visual inspection of the data showed similar results in both groups from P49 to P70. Bonferroni corrections were used to adjust for multiple comparisons in the behavioral testing data. For quantitative IHC analyses, the ratio of positive staining to total tissue area was calculated for each animal, and the median value was calculated by treatment group and sex. Comparisons between injured animals and controls were performed using a Wilcoxon-Mann-Whitney U-test with the Bonferroni correction for multiple comparisons. The outcomes of reflex and catwalk testing were compared using Spearman's rank correlations. Statistical results with a $p$ value $<0.05$ were considered statistically significant.

\section{Results}

\section{Model Outcomes}

Eight litters including 60 kits were initially included, with an insult mortality of $18.3 \%(n=11)$. A subset of 15 animals (3 controls and 12 injured) was used for early time point studies to develop the model (data not shown). As a result, 34 animals (14 controls and 20 injured) survived to P70 and were included in the final analysis. The median (range) temperature of 7 nesting sentinel LPStreated animals $4 \mathrm{~h}$ after injection (immediately before hypoxia) was $34.7^{\circ} \mathrm{C}\left(34.3-37.1^{\circ} \mathrm{C}\right)$, which was similar to littermate controls $\left(35.1^{\circ} \mathrm{C}, 34.2-36.1^{\circ} \mathrm{C} ; n=8\right)$. On the day of the insult (P10), mean (SD) weight of the kits was 41.4 (8.8) $\mathrm{g}$ for males and 38.8 (7.2) $\mathrm{g}$ for females. Injured animals lost, on average, $8.5 \%(10.4 \%)$ body weight be- 


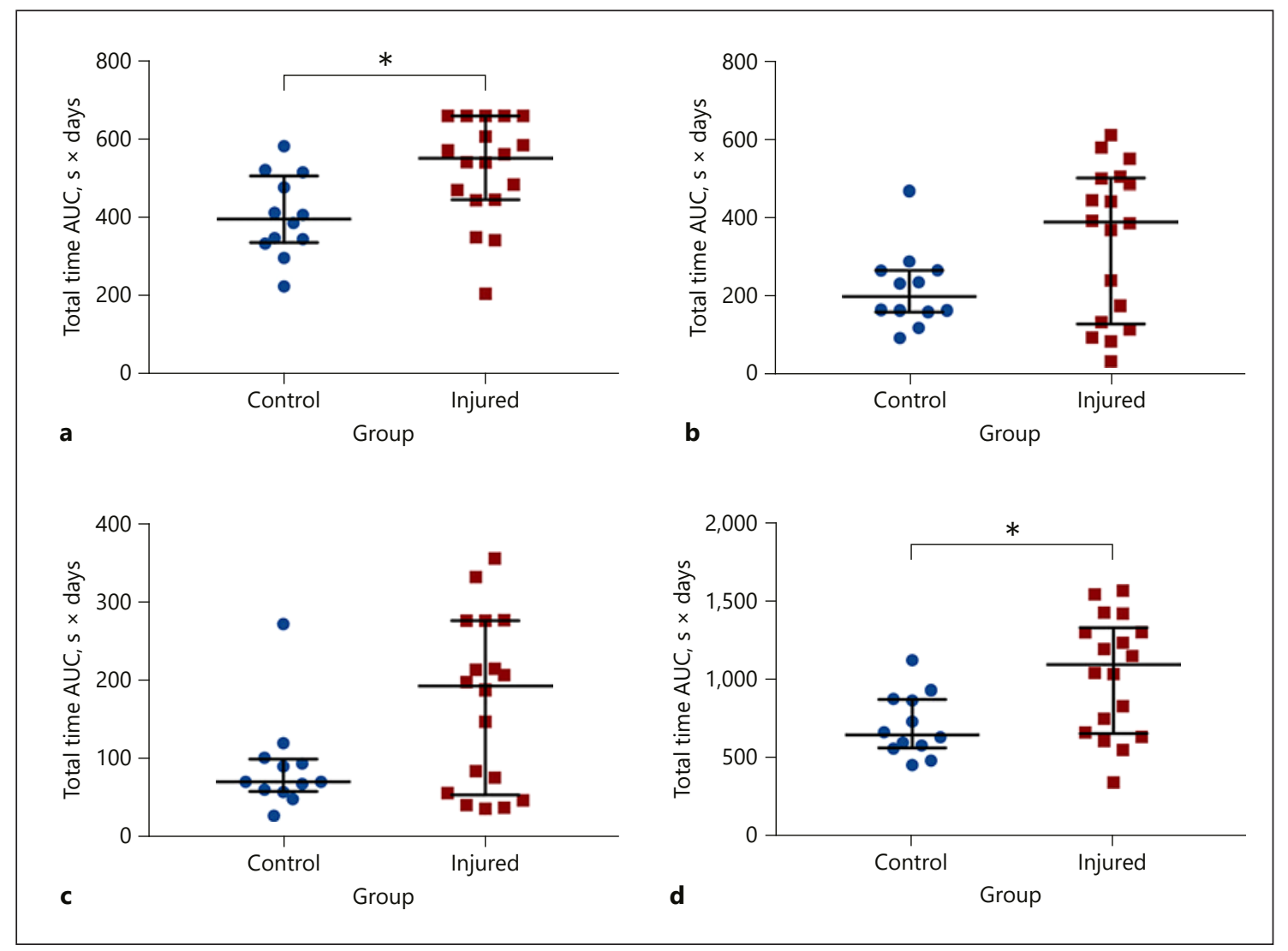

Fig. 2. Reflex development. Area under the curve (AUC) analysis for reflex development of negative geotaxis (a), cliff aversion (b), righting reflex (c), and total time across all 3 tests (d). Median (IQR) negative geotaxis was significantly slower to develop in the injured group than in the control group. No difference was seen in the AUC for cliff aversion or righting reflex. However, total time
AUC was significantly greater in the injured group compared to the control group. The pattern of the AUC results suggested a bimodal distribution of injury, with $6 / 18$ animals scoring similarly to control animals, and 12 displaying delayed skill development suggestive of cerebral injury. ${ }^{*} p<0.05$. tween $\mathrm{P} 10$ and $\mathrm{P} 11$ as a result of the insult. By P12, injured animals had gained $9.2 \%(13.7 \%)$ of their P10 body weight versus $24.4 \%(5.2 \%)$ in controls. By week 6 (P35-P42), injured animals had caught up to control animals in terms of weight, and males began to become heavier than females (Fig. 1). No difference in weight between injured and control animals was seen at P70.

\section{Early Reflex Testing}

At all ages (P28-P40), median time to complete all tasks (NG, CA, RR, and TT) was longer in the injured group than in controls; however, the variability in scores was high and differences were not consistently statistically significant. In all of the individual reflex tests, and in the TT score, median time was slower in the injured group than in the control group on every test day; how- ever, no significant difference was seen between groups in any given test at any age.

Analysis of the reflex AUCs showed that NG (median; IQR) was significantly slower to develop in the injured group (552 $\mathrm{s} \cdot$ days; 446-660 $\mathrm{s} \cdot$ days) than in the control group (397 $\mathrm{s} \bullet$ days; 337-507 $\mathrm{s} \bullet$ days) ( $p=0.03$; Fig. 2a). No difference was seen in the AUC for CA (Fig. 2b) or RR (Fig. 2c). However, TT AUC was significantly greater in the injured group $(1,101 \mathrm{~s} \cdot$ days; $663.5-1,338 \mathrm{~s} \cdot$ days $)$ than in the control group (656.4 s • days; $572.9-883.1 \mathrm{~s} \bullet$ days) ( $p=0.048)$. The pattern of the AUC results for CA, $\mathrm{RR}$, and TT suggest a bimodal distribution of injury, with 6/18 animals scoring similarly to control animals (Fig. 2), and 12 displaying delayed skill development suggestive of cerebral injury. No difference between injured male and female animals was seen at any time point. 


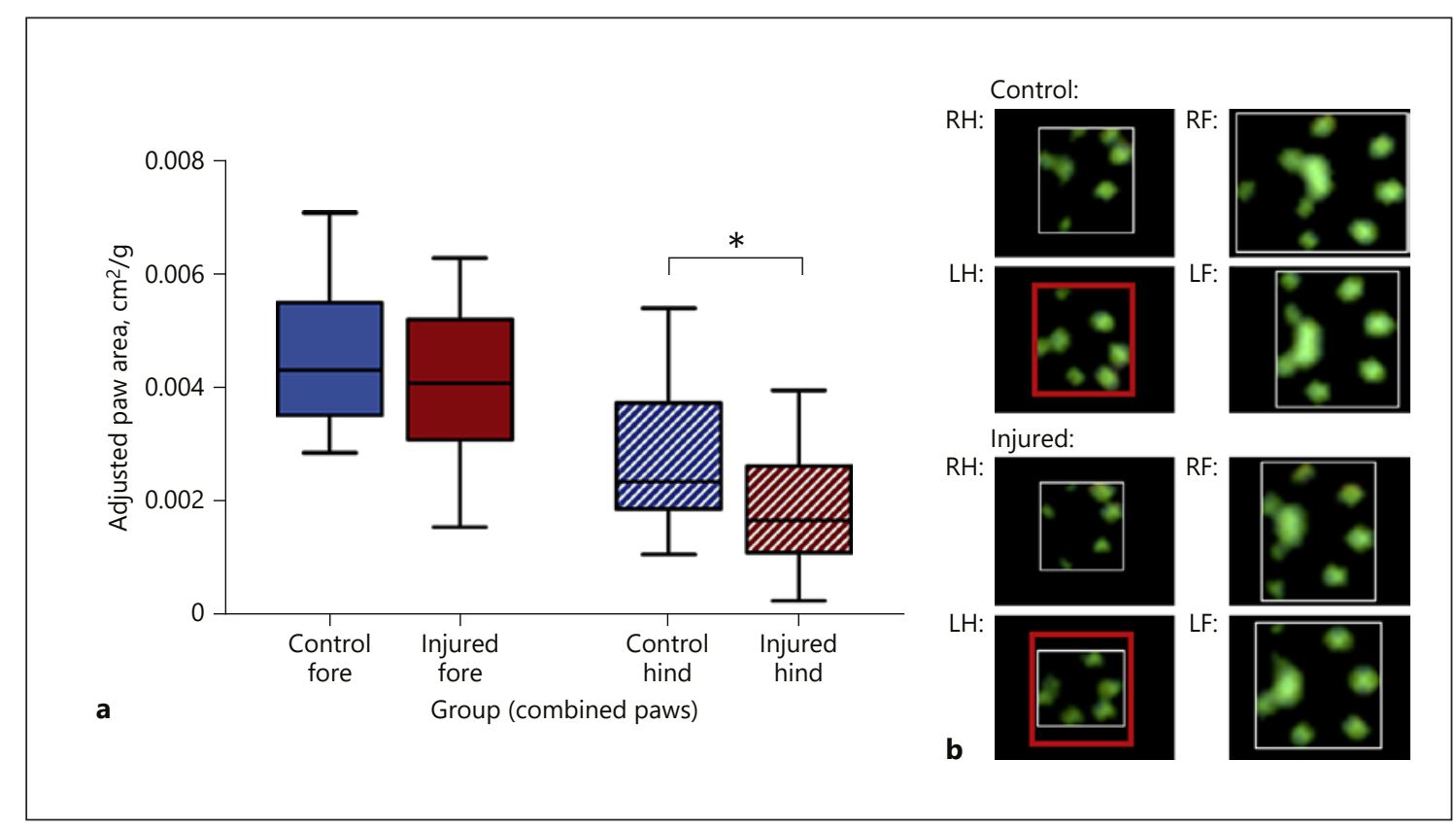

Fig. 3. Weight-adjusted paw areas. a At P42, adjusted area of the hind paws ( $\mathrm{RH}$ and $\mathrm{LH}$ ) of injured animals was significantly smaller than that of control animals, with no difference between forepaws (RF and LF). b Representative paw prints from the CatWalk software show that the hind paws of injured animals have a smaller area than the control animals. The red box around the LH print is the same size, for comparison. ${ }^{*} p<0.05$.

\section{Catwalk}

The greatest difference between the groups was seen at P42, with many deficits improving from P49 onwards. On P42, the weight-adjusted area (median; IQR) of the hind paws of injured animals $\left(1.68 \mathrm{~cm}^{2} / \mathrm{kg} ; 1.11-2.64 \mathrm{~cm}^{2} / \mathrm{kg}\right)$ was significantly smaller than that of control animals $\left(2.36 \mathrm{~cm}^{2} / \mathrm{kg} ; 1.88-3.75 \mathrm{~cm}^{2} / \mathrm{kg}\right)(p=0.05$; Fig. 3$)$. Similarly, this resulted in a larger ratio of forepaw and hind paw areas in injured animals $(2.34 ; 1.80-2.73)$ than in controls $(1.92 ; 1.39-2.29)(p=0.04)$. Intensity of pressure per unit area was also higher in the hind paws of injured animals at P42 $\left(89.8 \mathrm{au} / \mathrm{cm}^{2} ; 53.2-149.1 \mathrm{au} / \mathrm{cm}^{2}\right)$ than in control animals $\left(50.1 \mathrm{au} / \mathrm{cm}^{2} ; 37.9-81.3 \mathrm{au} / \mathrm{cm}^{2}\right)(p=$ 0.04; Fig. 4a). At P42, the BOS of the forepaws (relative to hind paws) was significantly wider in injured $(0.83 ; 0.67-$ $1.03)$ animals than in control animals $(0.62 ; 0.56-0.76)$ ( $p=0.009$; Fig. 4b). However, the wider BOS was not associated with any significant differences in measures of ataxic gait patterns (couplings and phase dispersions; data not shown). Instead, the gait of injured animals was characterized by an inability to consistently maintain forward motion in a straight line (online suppl. Video 1) when compared to controls (online suppl. Video 2). As measurements of lateral movement are not as well-de- fined in the CatWalk XT software, a custom Python package (FerretFit) was developed to analyze these movements from the paw print images extracted from CatWalk. Regarding paw print range, the lateral spread of prints relative to the midline was not significantly different between groups at any time point. However, the AUC (median; IQR) across the entire testing period was significantly greater in injured animals (1,786 pixels • days; $1,713-1,854$ pixels $\bullet$ days) than in control animals $(1,659$ pixels - days; $1,609-1,734$ pixels $\cdot$ days $)(p=0.008$, Fig. 4c). Using the same software package, a sine curve was fitted to the extracted paw prints from each run, and amplitudes were compared. There was a trend towards a greater amplitude (median; IQR) in the injured group (847.9 pixels $\bullet$ days; 660.3-963.7 pixels $\cdot$ days) than in the control group (690.5 pixels • days; 619.9-854.0 pixels - days) ( $p=0.1$; Fig. $4 \mathrm{~d})$. Figure 5 depicts the method of image processing by the software package. To see whether early reflex performance predicted performance on the catwalk, reflex TT AUC was compared to the catwalk parameters that were significantly different in injured animals at P42. Reflex TT AUC was significantly correlated with P42 adjusted hind paw area $(p=0.004$, $r=-0.64)$, forepaw-to-hind paw area ratio $(p=0.004, r=$ 


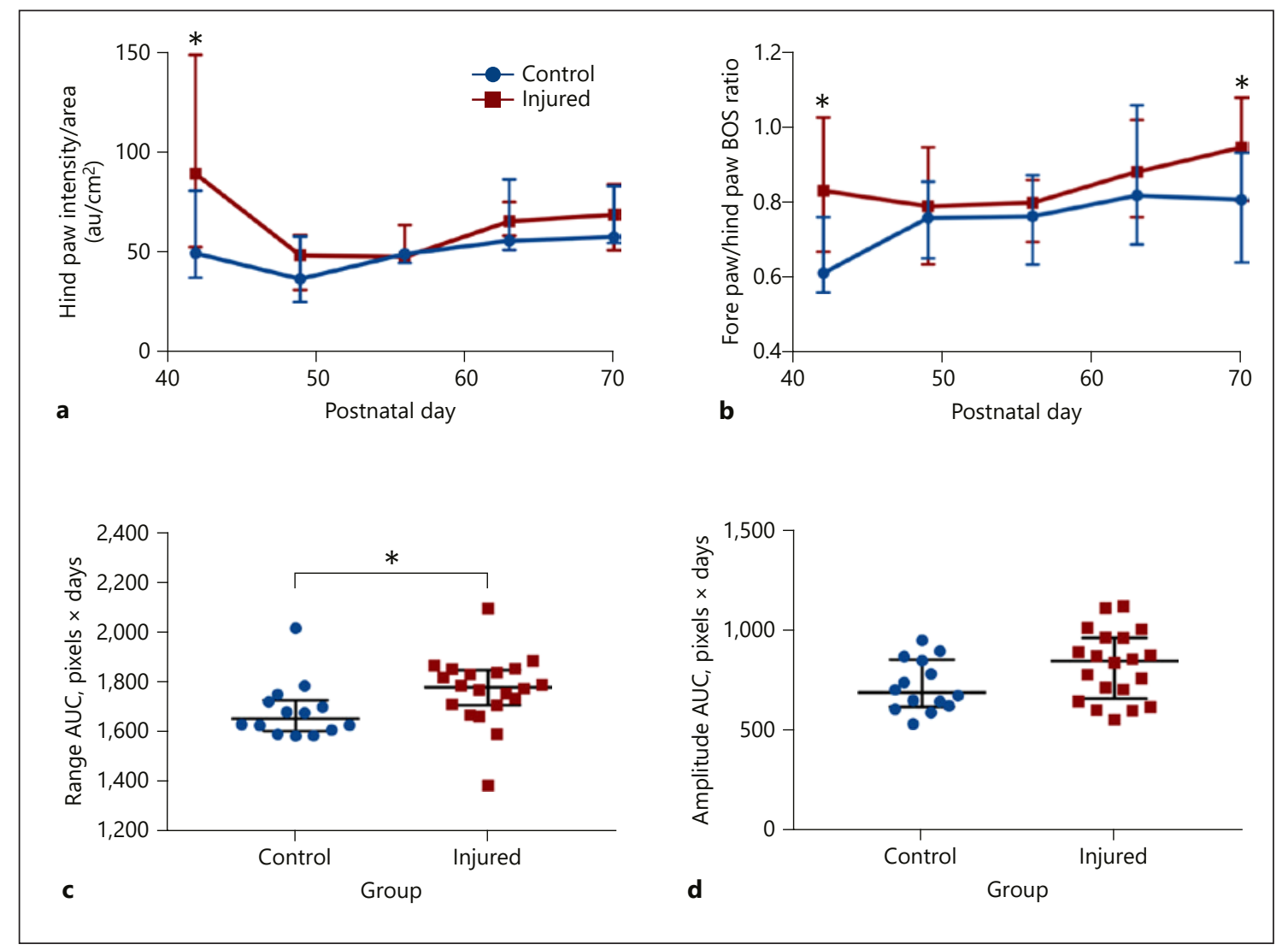

Fig. 4. Gait differences over time. Intensity of pressure per unit area was higher in the hind paws of injured animals than controls at P42 (a), and the base of support (BOS) ratio of the forepaws relative to the hind paws was also significantly wider in injured animals (b). However, these differences were absent in subsequent weeks of testing. c Using a custom Python package to analyze paw print trajectories, paw print range AUC across the entire testing period was significantly greater in injured animals than in control animals. d In the injured group, a trend towards a greater median path amplitude than in the control group was seen. ${ }^{*} p<0.05$.
$0.65)$, hind paw mean pressure intensity per unit area ( $p=0.003, r=0.66$ ), but not with BOS. No difference between injured male and female animals was seen at any time point.

\section{MRI and Connectome}

Greater FA values were seen in the control group in the right IC dorsolateral to the ventricle at the level of the thalamus (Fig. 6a). On T2-weighted imaging, significantly greater signal intensity was seen in the injured group throughout the white matter bilaterally (Fig. 6b). Network connectivity analysis showed 3/71 ROIs that were significantly different between injured and control animals (Fig. 6c, d). In ROI 3 (the right IC at the level of the mesencephalon), connectivity (mean; standard error) was significantly greater in injured females $(0.13 \mathrm{au} ; 0.015$ $\mathrm{au})$ than in control females (0.088 au; $0.018 \mathrm{au})(p=0.03)$. In the same ROI, connectivity in injured males ( $0.14 \mathrm{au}$;
$0.010 \mathrm{au})$ was significantly decreased compared to in control males ( $0.18 \mathrm{au} ; 0.007 \mathrm{au})(p=0.008)$. In ROI 19 (the left IC and associated white matter at the level of the caudate nucleus), connectivity was significantly greater in injured males ( $0.14 \mathrm{au} ; 0.006 \mathrm{au})$ than in control males $(0.10$ au; $0.009 \mathrm{au}$ ) ( $p=0.02)$. Similarly, in ROI 20 (the left IC and associated white matter at the level of the caudate nucleus, ventral to ROI 19), connectivity was significantly greater in in injured females $(0.11 \mathrm{au} ; 0.003 \mathrm{au})$ than in control females ( $0.09 \mathrm{au} ; 0.002 \mathrm{au})(p=0.003)$. Volumetric analysis from MRI outputs showed a trend towards greater cerebral volumes in control animals than in injured animals ( $p=0.09$; Fig. 6e). In the 9 injured females, the cerebral volume (median; IQR) was significantly decreased $\left(5.61 \mathrm{~cm}^{3} ; 5.46-6.35 \mathrm{~cm}^{3}\right)$ compared to in the 8 control females $\left(6.53 \mathrm{~cm}^{3} ; 6.23-6.94 \mathrm{~cm}^{3}\right)(p=0.002)$. Cerebral volume was not significantly different in the 10 injured $\left(7.06 \mathrm{~cm}^{3} ; 6.30-8.06 \mathrm{~cm}^{3}\right)$ and 6 control $\left(7.56 \mathrm{~cm}^{3}\right.$; 
Fig. 5. FerretFit paw print analysis. a For each catwalk run, an image showing every paw print was manually extracted from the CatWalk XT software. Using a specially developed ImageJ macro, the paw print boxes were identified and separated out (b) before being analyzed using the FerretFit Python library (c) to determine the total range of paw prints, as well as the amplitude of a sine curve that best fit the trajectory of the prints. RF, right forepaw; LF, left forepaw; $\mathrm{RH}$, right hind paw; LH, left hind paw.

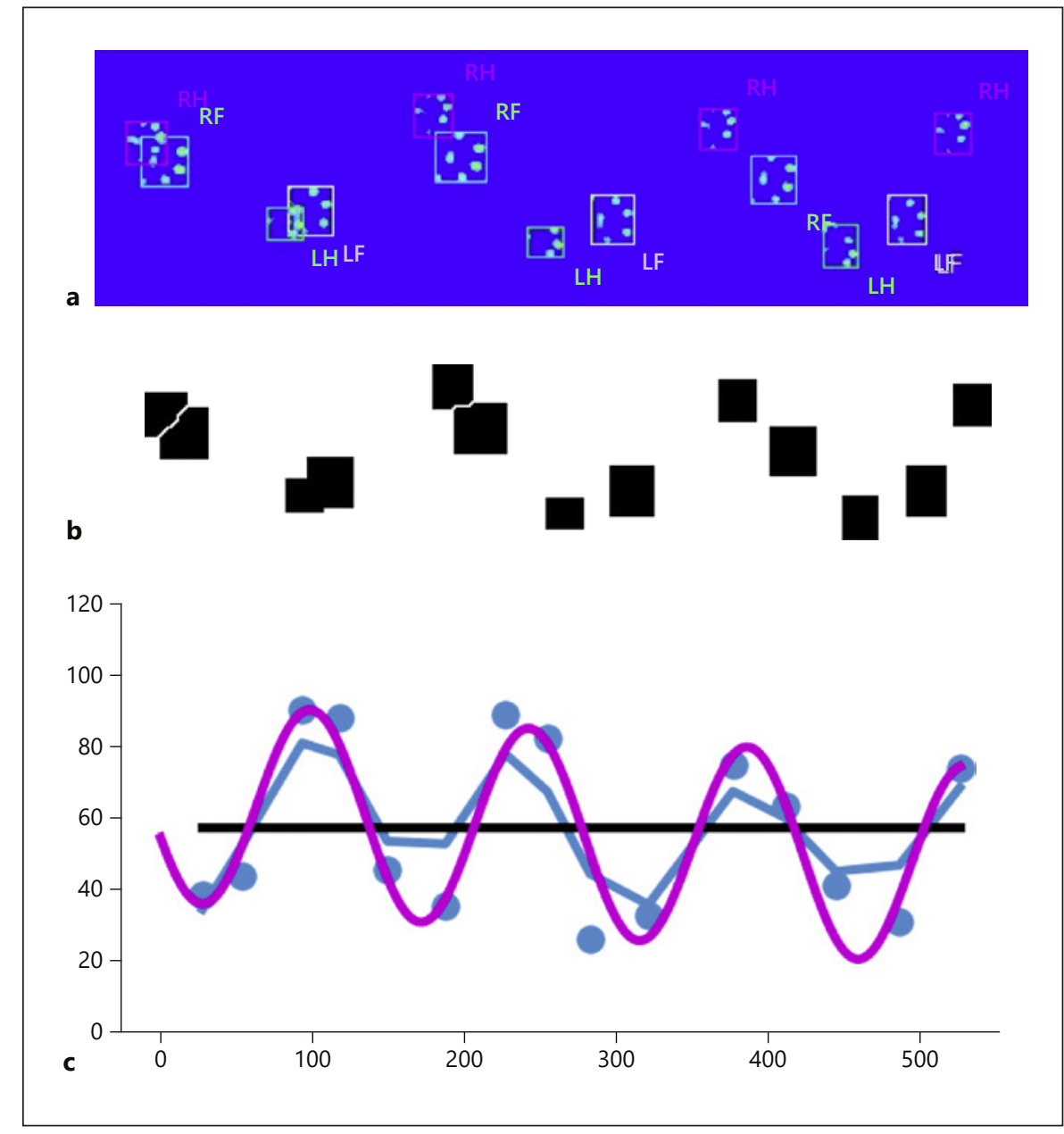

$\left.7.44-8.00 \mathrm{~cm}^{3}\right)$ males $(p=0.26)$. No difference in cerebral volumes was seen between injured and control animals (data not shown).

\section{Immunohistochemistry}

On IHC, MBP staining across an entire hemisphere as well as within the CC, was not significantly different between the 2 groups (data not shown). However, across 2 ROIs within the IC, the MBP staining ratio (median; IQR) was significantly greater and less variable in the injured group $(0.92 ; 0.90-0.9)$ than in the control group $(0.88 ; 0.84-0.93)(p=0.04)$. This was particularly evident in male animals (Fig. 7a). The thicknesses of the CC and $3 \mathrm{IC}$ areas at the base of consecutive sulci were then measured. Though no significant differences in thickness were seen within any individual region, a summary score based on the ranked weight-adjusted thickness of all 4 areas (a thinner IC or CC resulted in a lower rank) sug- gested thinning of the white matter in injured males $(p=$ 0.026; Fig. 7b). By comparison, OLIG2 staining intensity was decreased in the CC of injured animals $(0.042 ; 0.032-$ $0.046)$ compared to control animals $(0.048 ; 0.042-0.053)$ ( $p=0.033$; Fig. 7c). Across 2 ROIs within the IC, OLIG2 staining ratio was also lower in injured males $(0.031$; $0.028-0.040)$ compared to in control males $(0.037 ; 0.030$ $0.040)(p<0.05)$, but differences between injured and control females were not significant (Fig. $7 d$ ). Figure 8 shows images taken from animals best representing median MBP thickness and OLIG2 staining for both control and injured animals. No differences in GFAP staining intensity were seen either globally or regionally (in the CC, IC, and corona radiata). H\&E images at the level of the caudate nucleus and thalamus did not show substantial or consistent abnormalities in control or injured animals. 


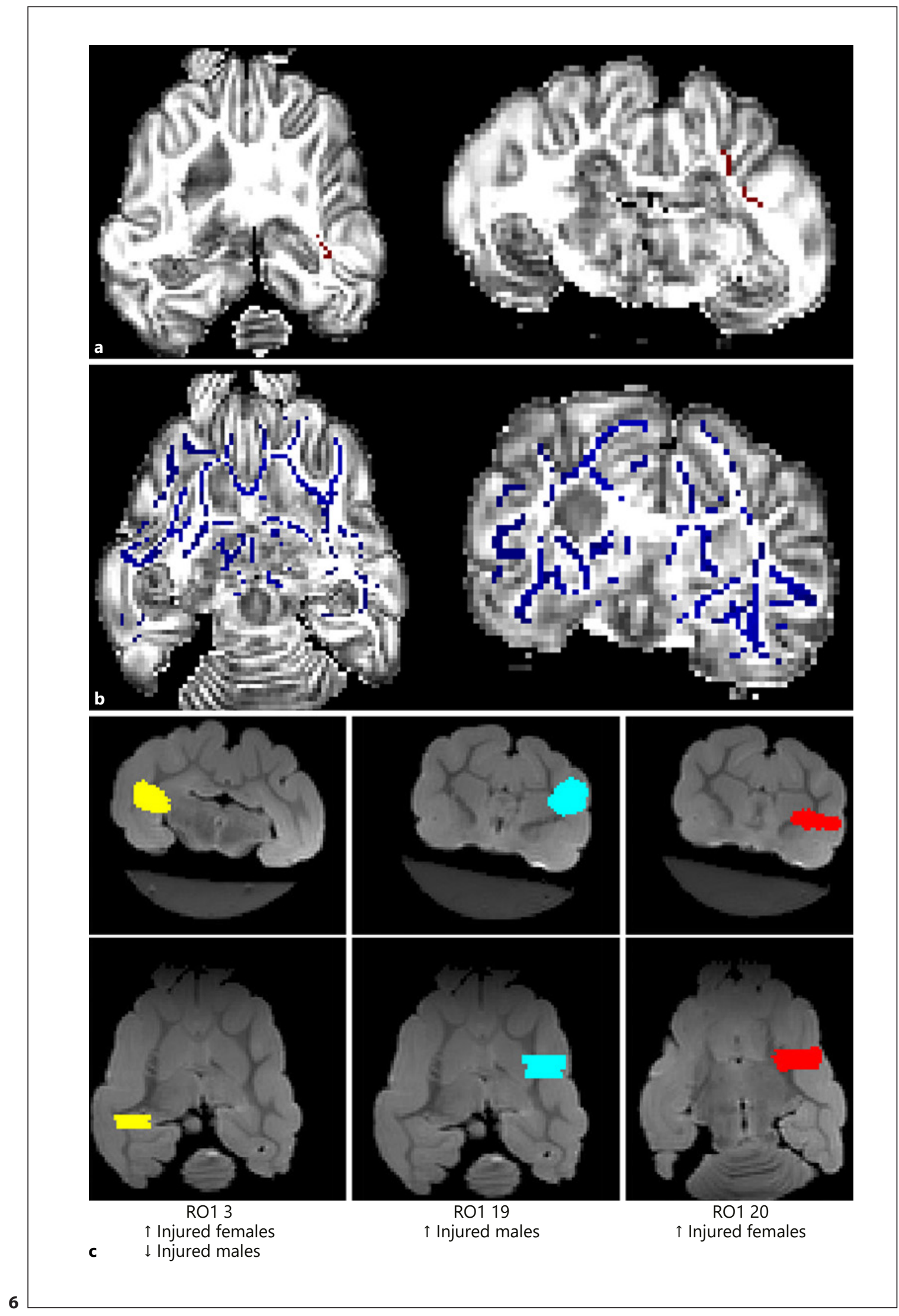

(Figure continued on next page.) 

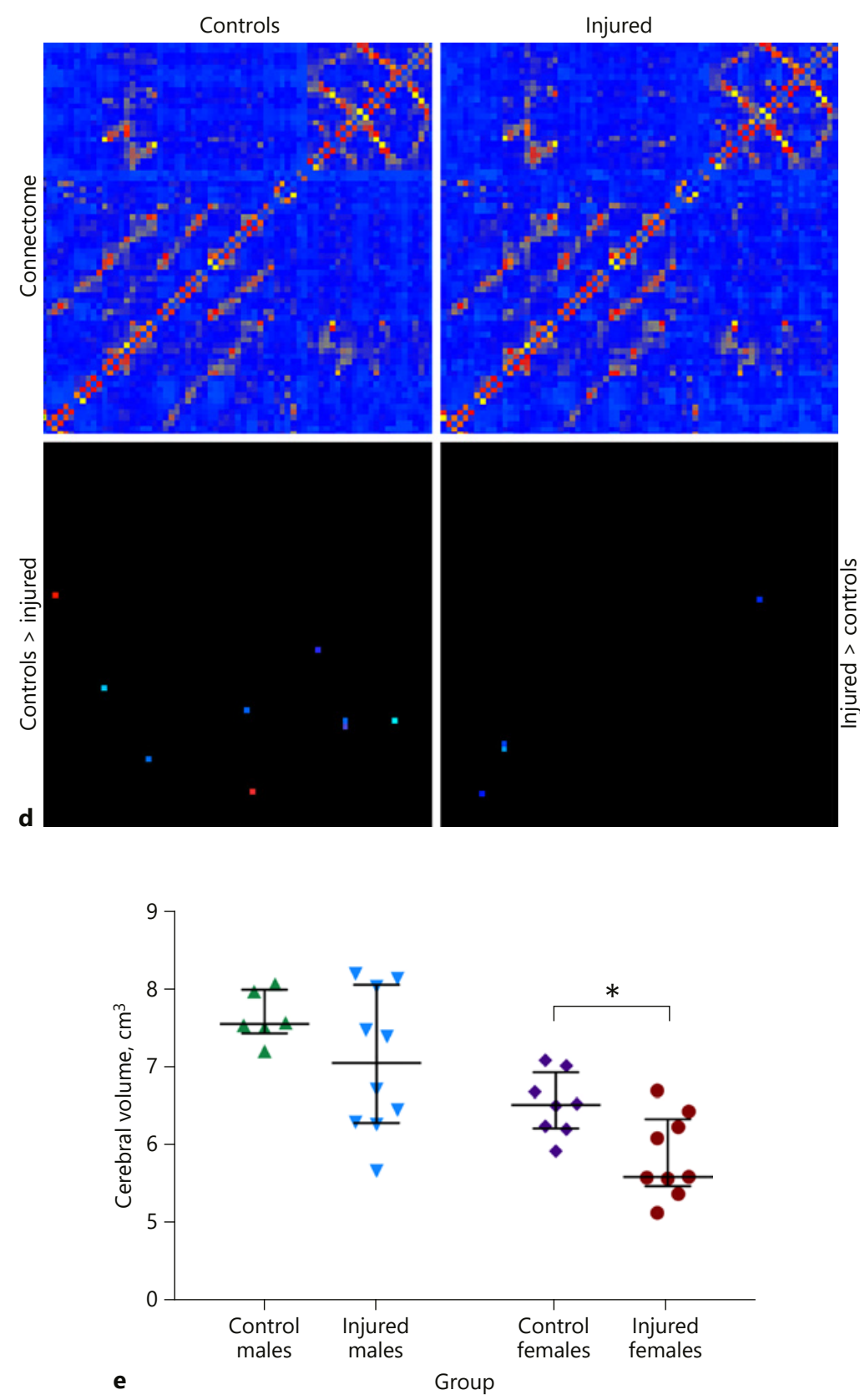

Fig. 6. MRI and connectome. a Greater fractional anisotropy values were seen in the control group in the right internal capsule (IC) dorsolateral to the ventricle at the level of thalamus (marked in red). b On T2-weighted imaging, significantly greater signal intensity was seen in the injured group throughout the white matter bilaterally (marked in blue). c Network connectivity analysis showed 3/71 ROIs that were significantly different between injured and control animals. In ROI 3 (right IC at the level of the mesencephalon), connectivity was greater in injured females than in control females. In the same ROI, connectivity in injured males was significantly decreased compared to in control males. In ROI 19 (left IC and associated white matter at the level of the caudate nucleus), mean connectivity was greater in injured males than in control males. In ROI 20 (left IC and associated white matter at the level of the caudate nucleus, ventral to ROI 19), mean connectivity was greater in injured females than in control females. d Overall connectivity projections show control (left panels) and injured (right panels) animals, with points of increased connectivity in controls compared to injured animals (bottom left panel), and increased connectivity in injured compared to control animals (bottom right panel). e Cerebral volumes in injured females were significantly decreased compared to control females, but no difference in cerebral volume was seen between injured and control males. ${ }^{*} p<0.05$. 


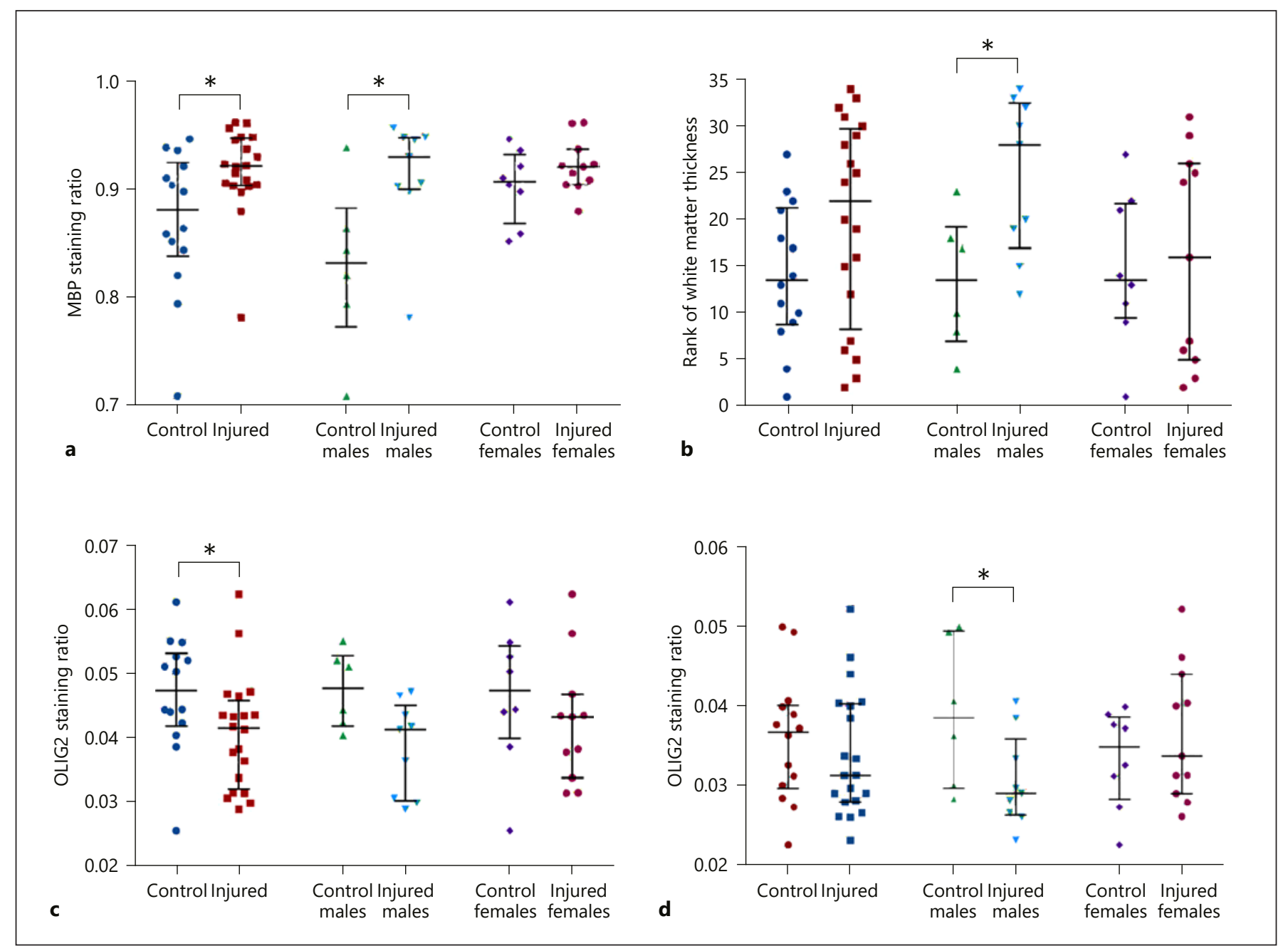

Fig. 7. Quantitative immunohistochemistry. a Within the internal capsule (IC), MBP staining ratio was significantly greater and less variable in the injured group than in the control group. This was particularly evident in male animals. $\mathbf{b}$ The thickness of the corpus callosum (CC) and 3 areas of the IC at the base of consecutive sulci were then measured, and a summary score based on the

\section{Discussion}

We present here a novel model of encephalopathy of prematurity in an extremely preterm-equivalent ferret. Compared to control animals, injured ferrets displayed delayed reflex development and early gait characteristics consistent with white-matter injury (WMI). At P70, an early childhood-equivalent age (i.e., roughly equivalent to a child 4-6 years of age), widespread white matter changes were seen on MRI and histology, including altered cerebral network connectivity and evidence of possible dysmaturation on IHC. ranked weight-adjusted thickness of all 4 areas suggested thinning of the white matter in injured males. c OLIG2 staining intensity was decreased in the CC of injured animals compared to in control animals. d OLIG2 staining ratio was also lower in injured males than in control males within the IC. $* p<0.05$.

There remains a significant need for an expanded repertoire of relevant large-animal models in which to test therapeutic interventions for infants with the neurological sequelae of prematurity. Rodent models often fail to recapitulate the WMI that is pathognomonic of premature brain injury, and few large-animal models of prematurity are available due to the relative brain development of model species at birth [27]. This is particularly evident when comparing the literature on premature brain injury to term hypoxic-ischemic encephalopathy, where a wide range of models allow for the sequential translation of therapies from in vitro work to rodent models and then

Dev Neurosci 2018;40:475-489 
Fig. 8. MBP and OLIG2 immunohistochemistry (IHC). Images are taken from animals best representing median MBP thickness and OLIG2 staining for both control and injured animals. Anti-myelin basic protein (MBP) IHC at the level of caudate nucleus shows 2 areas of the internal capsule (IC): IC1 in a control animal (a) and an injured animal (b), and IC2 in a control (c) and treated animal (d). $\times 5$. Positive anti-MBP staining (brown); hematoxylin counterstain (blue). Anti-OLIG2 IHC at the level of the IC in a control animal (e) and an injured animal $(\mathbf{f}) . \times 20$. Positive anti-OLIG2 staining (brown); hematoxylin counterstain (blue).

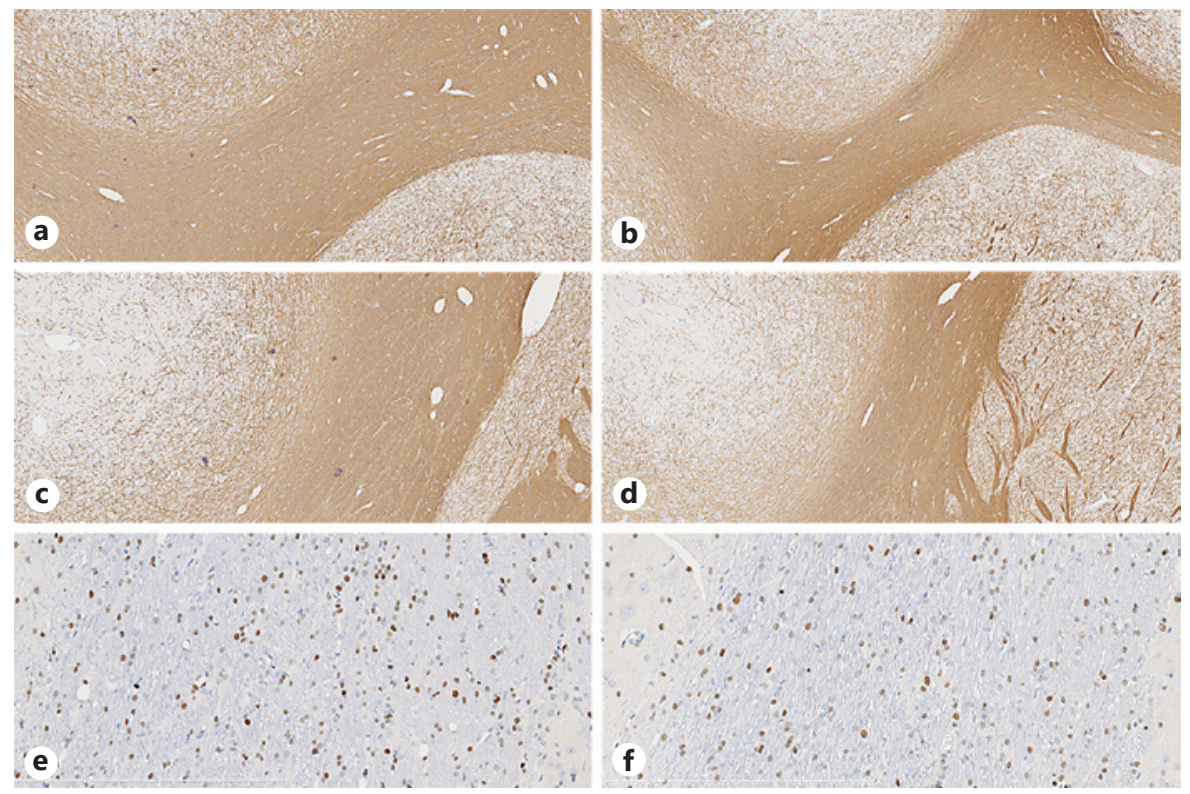

large-animal models before their application in clinical trials. This approach led to therapeutic hypothermia becoming the standard of care for infants with hypoxicischemic encephalopathy, as well as the development of pharmacological agents such as erythropoietin and xenon [28-32]. Our work in the newborn ferret aims to address some of this gap in the preclinical literature of premature brain injury.

The ferret is an attractive species in which to model premature brain injury due to its altricial nature. At birth, ferret cerebral development is equivalent to the human brain towards the end of the first trimester, with the P10 and P21 ferret equivalent to 24-26 weeks' gestation and term, respectively. This allows for the potential to model premature brain injury along the entire spectrum of ages seen clinically. Additionally, the ferret is amenable to long-term behavioral testing methods similar to those used in rodents [11]. This allows for the analysis of more complex and long-term behavioral outcome data than is available for other larger model animals such as piglets and sheep. However, one interesting aspect of developing preclinical models in the newborn ferret is its relative resistance to brain injury. During the development of the model, it became clear that standard injury mechanisms used in rodents (including presensitization with LPS, hypoxia, hyperoxia, and unilateral carotid artery ligation) did not lead to significant long-term brain injury in ferrets unless applied in specific combinations. Important variables include maintaining core temperature at $\geq 37^{\circ} \mathrm{C}$ during hypoxia, ensuring adequate hypoxia without producing significant intrahypoxic mortality, and moderating the oxygen concentration during hyperoxia; a hyperoxic oxygen concentration of $60 \%$ was used due to the fact that prolonged exposure to 80 or $100 \%$ oxygen after hypoxia resulted in acute pulmonary edema or hemorrhage. The final combination of LPS presensitization and alternating hypoxia/hyperoxia/hypoxia presented here results in a relatively mild but sustained injury detectable for up to 2 months after the initial injury.

In early reflex testing, injured animals showed slower median reflex times in every test on every day of testing. However, significant variability was seen within both groups, as well within individual animals across testing days, and these differences were not significantly different. Day-to-day variability in the performance of individual animals may be why individual comparisons were not different between groups. By comparison, AUC calculations of reflex development were significant between groups for NG and TT, perhaps because the accumulated differences over time became clear. This difference may have been due to delayed motor development in the injured animals, a delayed ability to learn the tasks, or a combination of the two. As with most small-animal models of neonatal brain injury, and indeed in premature infants seen clinically, there is a certain degree of variability seen in the injury that occurs as a result of the insult. This was particularly noticeable in the early reflex testing, where AUC calculations (as a measure of rate of reflex 
development over time) showed a distinct bimodal distribution of injury. Roughly two-thirds of animals displayed delayed reflex development, with the remaining third performing as well as control animals. Overall reflex development (TT AUC) was also significantly correlated with a number of catwalk markers associated with injury, suggesting that early reflex testing may be able to identify injured animals early on.

Based on our experience translating reflex testing to the newborn ferret, some degree of prior stimulation is required for the animal to engage with the tasks. It is likely that highly standardized pretesting procedures, as well as collecting a greater number of parameters during reflex testing, will allow for more granular and consistent differences between injured and control animals. This will form part of our future work with the model as a platform to test potential neuroprotective agents.

Gait assessment showed multiple deficits in the injured animals compared to controls, particularly at the earliest time point (P42). This included a widened base of support in the forepaws, which appeared to be an artifact of an unstable gait characterized by significant lateral movement. At P42, injured animals displayed smaller paw print areas in the hind paws, with the pad of the paw often absent from the visible paw print, both of which are suggestive of the toe-walking gait characteristic of spastic diplegia noted in preterm infants (Fig. 3a) [33]. This is also described in rodent models of WMI [34]. Toe-walking was associated with a greater relative intensity of paw placement in the hind paws during locomotion, as well as greater lateral movement during locomotion (measured as range and amplitude of paw prints) over the entire testing period. Despite the initial differences in gait and paw placement in injured animals at $\mathrm{P} 42$, the majority of these deficits disappeared from $\mathrm{P} 49$ onwards. This may be due to the fact that the ferret is able to adapt in spite of ongoing macroscopic brain injury. Similar results have been seen in a controlled cortical impact (CCI) model of traumatic brain injury in the adult male ferret [9]. Additionally, as the ferrets remained housed with the littermates during the survival period, natural periods of play within the nest may have constituted some degree of environmental enrichment, which is known to have a neuroprotective benefit [35].

Despite the variability and resolution of certain behavioral deficits, sustained WMI was seen throughout the white matter on both MRI and IHC. Ex vivo MRI showed extensive differences in white-matter signal on T2 imaging, especially in male animals. On IHC, this translated to a greater intensity of MBP staining within the IC, as well as relative thinning of the white matter in the $\mathrm{CC}$ and IC. At the same time, OLIG2 staining within the CC of injured animals, and the IC of injured males, was decreased compared to controls. Overall, this suggests a degree of sexually dimorphic white-matter dysmaturation after the insult. In males particularly, the insult resulted in the loss of OLIG2-positive cells and reduced white-matter thickness. The remaining functional oligodendrocytes then appeared to undergo accelerated myelination, with greater MBP density in the thinned white matter. However, it must be noted that white-matter thickness was only assessed immunohistochemically at a single level (at the caudate nucleus), and minor section-to-section variability during trimming and processing may contribute to variations in the apparent thickness of the white-matter tracts on 2D images. We were also unable to ascertain whether the increased MBP intensity in male animals was associated with normal MBP structure, or the mechanisms underlying the final observed histopathology. Therefore, future studies will include in-depth examination of white-matter structure, including electron microscopy, as well as examination of the time course of the expression of white-matter markers after injury, and how this differs between the sexes.

Interestingly, and consistent with the resilience and plasticity of the ferret brain after injury, connectome changes on MRI included increased connectivity in the IC in both males and females, perhaps as a response to deficits resulting from more widespread WMI. This was particularly evident in injured female animals, where multiple areas of increased connectivity were seen despite smaller overall brain volumes than control female animals. Though the brains of injured female animals showed fewer white-matter abnormalities than injured males, overall cerebral volume was decreased in injured females despite no difference in body weight from control females.

This work does have some additional limitations. Based on our parameters of LPS dosing and hypoxia/hyperoxia exposure, we were unable to determine exactly which factor(s) contributed most significantly to the final injury. However, based on our iterative approach to developing the model, it is likely that all factors played an important role in the final injury observed. We previously showed that LPS exposure results in microglial activation in the P10 ferret brain [11], but LPS alone did not appear to produce lasting injury. LPS exposure in nearterm-equivalent rodents resulted in a circulating inflammatory cytokine peak around $4 \mathrm{~h}$ after exposure, which corresponds with sensitization of the brain to hypoxia- 
ischemia and a significant increase in brain injury [1416]. A similar time course of inflammatory cytokine release (peak TNF- $\alpha$ and IL-6 release $2-4 \mathrm{~h}$ after LPS exposure) was seen in isolated ferret peripheral blood mononuclear cells [17]. This suggests that the ideal timing of LPS exposure for presensitization of the brain is likely to be similar in rodents and ferrets, and this was the final approach used here. However, our data do not allow for the comparison of the effects of hypoxia or hyperoxia alone without inflammatory presensitization.

Similar to the isolated effects of LPS and hypoxia, our work to date has not included multiple iterations of hyperoxia duration. As with any preclinical model, the goal was not to accurately reproduce the exposures encountered by preterm infants clinically, but to provide a confluence of the mechanistic factors thought to be involved in premature brain injury. These include inflammation, hypoxia, and the production of reactive oxygen species [7]. The last of these is exacerbated by hyperoxia, which is particularly problematic in premature infants due to a relatively underdeveloped antioxidative capacity $[36,37]$. As such, hyperoxia was included in the protocol to exacerbate the production of reactive oxygen species after a period of inflammatory activation and hypoxia. We used a prolonged period of hyperoxia compared to what might be seen clinically to maximize hyperoxia-induced oxidative stress and increase the likelihood of sustained cerebral injury.

With respect to future use of this model, a greater initial injury severity would be desirable, such that the effects of any therapeutic intervention would be easier to detect in behavioral outcomes as well as on MRI and pathology. Though no difference was seen between the sexes on any behavioral measure at any time point, those seen on MRI suggest that differences may be detectable with larger group sizes, more severe injury, or a greater battery of testing procedures. As with other models of neonatal brain injury, it is also likely that males and females will respond differently to any neuroprotective therapies. Future studies will involve further iteration of the model to increase injury severity, as well as increasing the number of behavioral tests to include cognition and other sensory assessments $[9,38]$. Mechanistic exploration of the ferret's resilience to brain injury may also allow for that resilience to be "reverse-engineered" as a way to develop potential therapeutic strategies for human preterm brain injury.

In summary, we present long-term characterization of the behavioral deficits, pathology, and changes in brain structure seen after an inflammation-sensitized hypoxic/ hyperoxic brain injury model in the P10 ferret. This newborn ferret model has the potential to provide an additional platform on which to assess potential therapies for encephalopathy in infants born extremely preterm.

\section{Acknowledgements}

The authors would like to acknowledge Brian Johnson, Juliet Hahn, and Megan Larmore at the University of Washington Histology and Imaging Core for their assistance in section preparation, IHC, whole-slide imaging, and quantitative image analysis. We also thank Vivienne Acuna, Simar Virk, Olivia White, and Alair Holden-Hunt for their assistance with animal handling and behavioral testing.

\section{Statement of Ethics}

The authors confirm that all animal experiments were performed in accordance with the ARRIVE (Animal Research: Reporting of In Vivo Experiments) guidelines and the NIH Guide for the Care and Use of Laboratory Animals and part of a protocol approved by the University of Washington Institutional Animal Care and Use Committee.

\section{Disclosure Statement}

The authors have no conflicts of interest to declare.

\section{Funding Sources}

This work was supported by NICHD grant No. 5R21NS093154.

\section{Author Contributions}

TW, DM, KC, PP, and SEJ designed and performed the experiments, and collected and analyzed the data. JSM performed the IHC and pathological assessments. CT prepared samples for MRI and analyzed and interpreted the results. CC and EN developed the software package for paw print analysis. TW drafted and revised the manuscript. All authors contributed to the critical evaluation and revision of the manuscript, and approved the final version. 


\section{References}

1 Martin JA, Hamilton BE, Osterman MJ, et al. Births: Final Data for 2016. National Vital Statistics Reports. US Dept of Health and Human Services Centers for Disease Control and Prevention, National Center for Health Statistics, NVSS. 2018 Jan;67(1):1-55.

2 Younge N, Goldstein RF, Bann CM, Hintz SR, Patel RM, Smith PB, et al; Eunice Kennedy Shriver National Institute of Child Health and Human Development Neonatal Research Network. Survival and Neurodevelopmental Outcomes among Periviable Infants. N Engl J Med. 2017 Feb;376(7):617-28.

3 Johnson S, Marlow N. Early and long-term outcome of infants born extremely preterm. Arch Dis Child. 2017 Jan;102(1):97-102.

4 Galinsky R, Polglase GR, Hooper SB, Black MJ, Moss TJ. The consequences of chorioamnionitis: preterm birth and effects on development. J Pregnancy. 2013;2013:412831.

5 Reich B, Hoeber D, Bendix I, FelderhoffMueser U. Hyperoxia and the Immature Brain. Dev Neurosci. 2016;38(5):311-30.

6 Bennet L, Dhillon S, Lear CA, van den Heuij L, King V, Dean JM, et al. Chronic inflammation and impaired development of the preterm brain. J Reprod Immunol. 2018 Feb;125: 45-55.

7 Galinsky R, Lear CA, Dean JM, Wassink G, Dhillon SK, Fraser M, et al. Complex interactions between hypoxia-ischemia and inflammation in preterm brain injury. Dev Med Child Neurol. 2018 Feb;60(2):126-33.

8 Empie K, Rangarajan V, Juul SE. Is the ferret a suitable species for studying perinatal brain injury? Int J Dev Neurosci. 2015 Oct;45:2-10.

9 Schwerin SC, Chatterjee M, Imam-Fulani AO, Radomski KL, Hutchinson EB, Pierpaoli $\mathrm{CM}$, et al. Progression of histopathological and behavioral abnormalities following mild traumatic brain injury in the male ferret. J Neurosci Res. 2018 Apr;96(4):556-72.

10 Rafaels KA, Bass CR, Panzer MB, Salzar RS, Woods WA, Feldman SH, et al. Brain injury risk from primary blast. J Trauma Acute Care Surg. 2012 Oct;73(4):895-901.

11 Snyder JM, Wood TR, Corry K, Moralejo DH, Parikh P, Juul SE. Ontogeny of white matter, toll-like receptor expression, and motor skills in the neonatal ferret. Int $\mathrm{J}$ Dev Neurosci. 2018 Nov;70:25-33.

12 Barnette AR, Neil JJ, Kroenke CD, et al. Characterization of Brain Development in the Ferret via Magnetic Resonance Imaging. Pediatr Res. 2009;66(1):80-4.

13 Noctor SC, Scholnicoff NJ, Juliano SL. Histogenesis of ferret somatosensory cortex. J Comp Neurol. 1997 Oct;387(2):179-93.

14 Eklind S, Mallard C, Leverin AL, Gilland E, Blomgren K, Mattsby-Baltzer I, et al. Bacte- rial endotoxin sensitizes the immature brain to hypoxic-ischaemic injury. Eur J Neurosci. 2001 Mar; 13(6):1101-6.

15 Falck M, Osredkar D, Wood TR, Maes E, Flatebø T, Sabir H, et al. Neonatal Systemic Inflammation Induces Inflammatory Reactions and Brain Apoptosis in a Pathogen-Specific Manner. Neonatology. 2018;113(3):21220.

16 Osredkar D, Sabir H, Falck M, Wood T, Maes E, Flatebø T, et al. Hypothermia Does Not Reverse Cellular Responses Caused by Lipopolysaccharide in Neonatal Hypoxic-Ischaemic Brain Injury. Dev Neurosci. 2015;37(4-5): 390-7.

17 Nakata M, Itou T, Sakai T. Quantitative analysis of inflammatory cytokines expression in peripheral blood mononuclear cells of the ferret (Mustela putorius furo) using real-time PCR. Vet Immunol Immunopathol. 2009 Jul; 130(1-2):88-91.

18 Rubinov M, Sporns O. Complex network measures of brain connectivity: uses and interpretations. Neuroimage. 2010 Sep;52(3): 1059-69.

19 Fair DA, Cohen AL, Dosenbach NU, Church JA, Miezin FM, Barch DM, et al. The maturing architecture of the brain's default network. Proc Natl Acad Sci USA. 2008 Mar; 105(10):4028-32.

20 Fair DA, Cohen AL, Power JD, et al. Functional brain networks develop from a "local to distributed" organization. PLoS Comput Biol. 2009;5(5):e1000381.

21 Fair DA, Dosenbach NU, Church JA, Cohen AL, Brahmbhatt S, Miezin FM, et al. Development of distinct control networks through segregation and integration. Proc Natl Acad Sci USA. 2007 Aug;104(33):13507-12.

22 Gong G, Rosa-Neto P, Carbonell F, Chen ZJ, $\mathrm{He} \mathrm{Y,} \mathrm{Evans} \mathrm{AC.} \mathrm{Age-} \mathrm{and} \mathrm{gender-related} \mathrm{dif-}$ ferences in the cortical anatomical network. J Neurosci. 2009 Dec;29(50):15684-93.

23 Meunier D, Achard S, Morcom A, Bullmore E. Age-related changes in modular organization of human brain functional networks. Neuroimage. 2009 Feb;44(3):715-23.

24 Buckner RL, Sepulcre J, Talukdar T, Krienen FM, Liu H, Hedden T, et al. Cortical hubs revealed by intrinsic functional connectivity: mapping, assessment of stability, and relation to Alzheimer's disease. J Neurosci. 2009 Feb; 29(6):1860-73

25 Liu Y, Liang M, Zhou Y, He Y, Hao Y, Song $\mathrm{M}$, et al. Disrupted small-world networks in schizophrenia. Brain. 2008 Apr;131(Pt 4): 945-61.

26 Wang L, Zhu C, He Y, Zang Y, Cao Q, Zhang $\mathrm{H}$, et al. Altered small-world brain functional networks in children with attention-deficit/ hyperactivity disorder. Hum Brain Mapp. 2009 Feb;30(2):638-49.

27 Dobbing J, Sands J. Comparative aspects of the brain growth spurt. Early Hum Dev. 1979 Mar;3(1):79-83.

28 McPherson RJ, Juul SE. Erythropoietin for infants with hypoxic-ischemic encephalopathy. Curr Opin Pediatr. 2010 Apr;22(2):139-45.

29 Traudt CM, McPherson RJ, Bauer LA, Richards TL, Burbacher TM, McAdams RM, et al. Concurrent erythropoietin and hypothermia treatment improve outcomes in a term nonhuman primate model of perinatal asphyxia. Dev Neurosci. 2013;35(6):491503

30 Chakkarapani E, Dingley J, Aquilina K, Osredkar D, Liu X, Thoresen M. Effects of xenon and hypothermia on cerebrovascular pressure reactivity in newborn global hypoxicischemic pig model. J Cereb Blood Flow Metab. 2013 Nov;33(11):1752-60.

31 Dingley J, Tooley J, Liu X, Scull-Brown E, Elstad M, Chakkarapani E, et al. Xenon ventilation during therapeutic hypothermia in neonatal encephalopathy: a feasibility study. Pediatrics. 2014 May;133(5):809-18.

32 Thoresen M, Hobbs CE, Wood T, Chakkarapani E, Dingley J. Cooling combined with immediate or delayed xenon inhalation provides equivalent long-term neuroprotection after neonatal hypoxia-ischemia. J Cereb Blood Flow Metab. 2009 Apr;29(4):707-14.

33 Zhou J, Butler EE, Rose J. Neurologic Correlates of Gait Abnormalities in Cerebral Palsy: implications for Treatment. Front Hum Neurosci. 2017 Mar;11:103.

34 Jantzie LL, Robinson S. Preclinical Models of Encephalopathy of Prematurity. Dev Neurosci. 2015;37(4-5):277-88.

35 Durán-Carabali LE, Arcego DM, Odorcyk FK, Reichert L, Cordeiro JL, Sanches EF, et al. Prenatal and Early Postnatal Environmental Enrichment Reduce Acute Cell Death and Prevent Neurodevelopment and Memory Impairments in Rats Submitted to Neonatal Hypoxia Ischemia. Mol Neurobiol. 2018 May; 55(5):3627-41.

36 Johnston MV, Fatemi A, Wilson MA, Northington F. Treatment advances in neonatal neuroprotection and neurointensive care. Lancet Neurol. 2011 Apr;10(4):372-82.

37 Sheldon RA, Aminoff A, Lee CL, Christen S, Ferriero DM. Hypoxic preconditioning reverses protection after neonatal hypoxia-ischemia in glutathione peroxidase transgenic murine brain. Pediatr Res. 2007 Jun;61(6): 666-70.

38 Garipis N, Hoffmann KP. Visual field defects in albino ferrets (Mustela putorius furo). Vision Res. 2003 Mar;43(7):793-800. 